Sílvia Maria Madeira Fernandes ${ }^{1}$

ANa Margarida Novals Maçães ${ }^{1}$ ANDREIA LEITÃo MARQUES ${ }^{2}$

Helena Vasconcelos Barros Leite ${ }^{3}$

Artigo Original

Palavras-chave

Histeroscopia/métodos Infertilidade feminina

Aborto habitual

Útero/anormalidades

Keywords

Hysteroscopy/methods Infertility, female

Abortion, habitual

Uterus/abnormalities

\title{
Desfecho reprodutivo após septoplastia histeroscópica: estudo retrospectivo
}

\author{
Reproductive outcomes after bysteroscopic septoplasty: \\ a retrospective study
}

\section{Resumo}

OBJETIVO: Avaliar os resultados reprodutivos após septoplastia histeroscópica. MÉTODOS: Estudo retrospetivo observacional por meio de análise dos registos clínicos de 28 mulheres com antecedentes de infertilidade ou de abortos recorrentes, submetidas à septoplastia histeroscópica. Para a avaliação do desfecho reprodutivo foram consultados os registos informáticos dos Cuidados de Saúde Primários e do nosso Centro Hospitalar, no período compreendido entre a septoplastia e a primeira gravidez ou o presente. Os outcomes primários foram a taxa de gravidez, de recém-nascidos e de abortos após septoplastia. O septo uterino foi diagnosticado por ecografia 2D ou 3D e classificado de acordo com a classificação da American Fertility Societr. A septoplastia foi realizada na fase folicular do ciclo menstrual, com recurso da energia monopolar, bipolar e/ou microtesoura. Os programas Microsoft Excel e SPSS versão 17 foram utilizados para comparação do desfecho reprodutivo prévio e posterior à septoplastia. Foi utilizado o teste exato de Fisher, considerando significado estatístico quando p<0,05. RESULTADOS: Foi realizada septoplastia histeroscópica em 20 doentes (72\%) com infertilidade secundária e 8 (28\%) com infertilidade primária, tendo sido necessária segunda intervenção para remoção completa do septo em 5 casos (18\%). Ocorreu um caso perfuração uterina minor. Após septoplastia histeroscópica, $64 \%$ das mulheres engravidaram, obtendo-se uma taxa de nados vivos de 48\%; gravidez tubária de 4\%; e 19\% das doentes tiveram abortos espontâneos. CONCLUSÕES: Os resultados do presente estudo estão de acordo com o descrito na literatura, tendo-se obtido uma melhoria significativa dos desfechos reprodutivos, com uma redução da taxa de aborto espontâneo de cinco vezes após a septoplastia histeroscópica.

\section{Abstract}

PURPOSE: To assess the reproductive outcomes after hysteroscopic septoplasty. METHODS: A retrospective observational study was performed with analysis of the medical records of 28 women with infertility or recurrent abortions undergoing hysteroscopic septoplasty. To evaluate reproductive outcomes we consulted the medical records of our hospital and of primary health care units between septoplasty and the present or first pregnancy. Primary outcomes were pregnancy rate, newborns, and abortions after septoplasty. Uterine septum was diagnosed by 2D or 3D ultrasound and classified according to the American Fertility Society. All procedures were performed in the follicular phase of the menstrual cycle using monopolar or bipolar energy and/or microscissors. To compare the reproductive outcomes before and after septoplasty we used Microsoft Excel and SPSS version 17. Fisher's exact test was considered statistically significant if $p<0.05$. RESULTS: Hysteroscopic septoplasty was performed in 20 patients (72\%) with secondary infertility and in 8 patients (28\%) with primary infertility. The septum was incompletely removed during the first hysteroscopy in 5 cases (18\%), which required a second surgery. One case was complicated with minor uterine perforation. After hysteroscopic septoplasty, $64 \%$ of women became pregnant and $48 \%$ live neonates were delivered; $4 \%$ of the patients had a tubal pregnancy; and 19\% had miscarriages. CONCLUSIONS: The results of this study are consistent with those described in the literature. Patients obtained a significant improvement of reproductive outcomes with a fivefold reduction in miscarriage rate after hysteroscopic septoplasty.

Correspondência

Silvia Maria Madeira Fernandes Serviço de Ginecologia, Maternidade Bissaya Barreto Rua Augusto $3000-061$ Coimbra, Portugal

Recebido

$29 / 12 / 2014$

Aceito com modificacōes

$26 / 01 / 2015$
Serviço de Ginecologia da Maternidade Bissaya Barreto, Centro Hospitalar e Universitário de Coimbra - Coimbra, Portugal. 'Serviço de Ginecologia, Maternidade Bissaya Barreto, Centro Hospitalar e Universitário de Coimbra - Coimbra, Portugal. ${ }^{2}$ Centro Hospitalar e Universitário de Coimbra - Coimbra, Portugal

${ }^{3}$ Serviço de Reprodução Humana e de Ginecologia, Centro Hospitalar e Universitário de Coimbra - Coimbra, Portugal.

Conflito de interesses: não há. 
Introdução

O útero septado é uma malformação uterina congênita que resulta da incompleta reabsorção dos ductos müllerianos ou para-mesonéfricos. Esses são formados a partir das seis semanas de desenvolvimento embrionário, com uma invaginação do epitélio celômico. Na sequência de um processo de migração medial e descendente, fundem-se para formar o canal uterovaginal, dividido longitudinalmente por um septo, cuja reabsorção irá perdurar até as $17^{\mathrm{a}}$ a $20^{\mathrm{a}}$ semanas ${ }^{1-3}$. A regulação molecular do desenvolvimento mülleriano é efetuada pelos genes da família HOX, sendo os genes HOXA 10 e HOXA 11 responsáveis pelo desenvolvimento do útero e os HOXA 11 e HOXA 13 pelo segmento inferior e colo ${ }^{4,5}$. O desenvolvimento dos canais de Müller e a reabsorção do septo uterino podem ser perturbados por múltiplos fatores, nomeadamente a exposição a disruptores endócrinos e a ausência de $\mathrm{B} c \mathrm{l}-2^{1,5}$.

As malformações uterinas congênitas afetam 2 a $3 \%$ da população, embora a prevalência varie de acordo com os diferentes estudos, grupos populacionais e métodos de diagnóstico utilizados ${ }^{6-8}$. O útero septado é a malformação uterina mais frequente e está associada à mais elevada incidência de resultados reprodutivos adversos. A sua prevalência na população geral é de 0,5 a $6,7 \%$; entre as mulheres com antecedente de infertilidade, 2,4 a 7,3\%; e nas com aborto recorrente entre 5 e $16,7 \%^{7}$. Segundo uma metanálise de 2014, a probabilidade de gravidez está significativamente diminuída nas mulheres com útero septado em relação àquelas do grupo de controle, sendo o risco relativo (RR) 0,8 e o intervalo de confiança de $95 \%$ (IC95\%) 0,77-0,96. Foi verificado ainda que as mulheres com septo uterino apresentam um risco mais elevado, comparativamente àquelas do grupo de controle, de aborto do primeiro trimestre e de apresentações fetais anômalas .

O diagnóstico de septo uterino assenta fundamentalmente na imagenologia, considerando como exames de maior acuidade a histeroscopia associada à laparoscopia, ecografia 3D, histerossonografia e histerossalpingografia (clássica e virtual) ${ }^{10,11}$.

As indicações formais para abordagem terapêutica dos septos uterinos são os quadros de aborto recorrente, podendo, contudo, ser extensível aos casos de antecedentes de aborto tardio, parto prematuro, infertilidade em nulíparas com mais de 35 anos, infertilidade inexplicada e previamente a técnicas de procriação medicamente assistida.

O presente estudo tem como propósito avaliar o desfecho reprodutivo após septoplastia por via histeroscópica em mulheres com infertilidade ou aborto recorrente.

\section{Métodos}

Foi realizado um estudo observacional retrospetivo com pacientes submetidas a septoplastia por via histeroscópica, entre janeiro de 2000 e dezembro de 2013. Foram incluídas no presente estudo 28 doentes com diagnóstico de útero septado, referenciadas por infertilidade e/ou antecedentes de abortos de repetição. O desfecho reprodutivo após a cirurgia foi conhecido por consulta dos registos informatizados dos Cuidados de Saúde Primários e do nosso Centro Hospitalar, no período compreendido entre a septoplastia e a primeira gravidez/presente. Os outomes primários foram a taxa de gravidez, de recém-nascidos e de abortos após septoplastia. Foram ainda avaliados a história ginecológica e obstétrica, classificação do tipo de septo uterino e as complicações cirúrgicas e pós-operatórias.

A septoplastia por via histeroscópica foi realizada em 8 (28\%) doentes com infertilidade primária com uma duração média de 3 31 ,9 anos (mínimo 1; máximo 7 anos) e em 20 com infertilidade secundária, com uma duração média de 3,5土3 anos (mínimo 1; máximo 15 anos). Em 14 doentes $(50 \%)$ havia antecedente de aborto do primeiro trimestre, sendo $6(43 \%)$ com 2 ou mais abortos consecutivos; 3 (11\%) tinham antecedente de aborto tardio; e 3 (11\%) tinham antecedente de parto pré-termo.

O diagnóstico de septo uterino foi realizado por ecografia 2D ou 3D em todas as doentes e em cinco delas por histerossalpingografia, no decurso da investigação etiológica da infertilidade do casal.

O tipo de septo uterino foi classificado de acordo com a classificação da American Fertility Society: 20 doentes (71\%) com septo parcial e 8 (29\%) com septo completo. Duas doentes apresentavam ainda septo vaginal associado.

Todos os procedimentos foram realizados durante a fase folicular do ciclo menstrual e sob anestesia geral. Em seis doentes foi utilizada energia monopolar e nas restantes o procedimento foi realizado com energia bipolar (ansa ou ponteira) e/ou microtesoura. Após entrar na cavidade endometrial e visualizar cada óstium tubar, foi realizada uma incisão equidistante entre as paredes anterior e posterior do útero, a partir da margem caudal do septo até o fundo uterino. A septoplastia foi considerada completa quando se obteve uma aparência normal da cavidade, visualizaram os dois ostia em simultâneo e se conseguia mobilizar o histeroscópio livremente na cavidade uterina.

A análise estatística foi efetuada com recurso do Microsoft Excel e SPSS versão 17. Foi utilizado o teste exato de Fisher para comparar frequências, considerando existir significado estatístico quando o $\mathrm{p}<0,05$.

\section{Resultados}

Foram incluídas no estudo 28 mulheres com diagnóstico de septo uterino com uma média de idade de $34 \pm 4$ anos. Das 8 mulheres com infertilidade primária, foram identificados fatores de infertilidade em 7 (88\%): anovulação (5); fator tubar (2); endometriose estadio II 
da American Society for Reproductive Medicine (ASRM) (1); e fator masculino (1). Das 20 mulheres com infertilidade secundária, 7 (35\%) tinham outros fatores de infertilidade associados: fator masculino (2); fator tubário (2); endometriose estadio IV da ASRM (2); miomas (2); e anovulação (1). $\mathrm{Na}$ Tabela 1 apresentamos as características clínicas das doentes submetidas à septoplastia histeroscópica.

Em 5 casos (18\%) houve necessidade de um segundo procedimento cirúrgico para remoção de septo residual: 1 dos quais em doente com septo parcial (5\%); e 4 em doentes com septo completo $(50 \%)$, com diferença estatisticamente significativa ( $\mathrm{p}=0,01$; teste exato de Fisher).

A septoplastia foi realizada isoladamente em 19 doentes $(68 \%)$, em 7 casos foi realizada concomitantemente laparoscopia diagnóstica; em 2 casos miomectomia abdominal; e em 1 exérese de septo vaginal. O tempo médio da cirurgia, quando isolada, foi $22 \pm 6$ minutos (mínimo 14; máximo 35 minutos). Dos 33 procedimentos realizados ocorreu um caso de perfuração uterina isolada (3\%).

Das 28 doentes submetidas à cirurgia, 2 não mantiveram o desejo de gravidez, e a doente em que ocorreu perfuração uterina optou por não ser submetida à septoplastia. Das restantes, 16 (64\%) engravidaram num período médio de 15 meses após o procedimento, 2 das quais após técnica de reprodução assistida. Das 16 doentes que engravidaram, 1 teve uma gravidez tubar (4\%), 3 tiveram aborto espontâneo precoce (19\%) e 12 tiveram partos com recém-nascidos vivos $(75 \%)$, um dos quais pré-termo às 36 semanas. $\mathrm{O}$ parto ocorreu por cesariana em 7 casos (58\%). Na Tabela 2 apresentamos os resultados reprodutivos após a septoplastia histeroscópica e a comparação dos resultados reprodutivos pré e pós-cirurgia.

Tabela 1. Caracterização da amostra e dos desfechos reprodutivos após septoplastia

\begin{tabular}{|c|c|c|c|}
\hline & \multicolumn{2}{|c|}{ Infertilidade } & \multirow{2}{*}{ Total } \\
\hline & Primária & Secundária & \\
\hline & n (\%) & $n(\%)$ & n (\%) \\
\hline Doentes & 8 & 20 & 28 \\
\hline Idade (anos) (média \pm DP) & $36 \pm 2$ & $33 \pm 5$ & $34 \pm 4$ \\
\hline \multicolumn{4}{|l|}{ Tipo de septo } \\
\hline Parcial & 7 & 13 & 20 \\
\hline Completo & 1 & 7 & 8 \\
\hline $\begin{array}{l}\text { Duração infertilidade (anos) } \\
\text { (média } \pm \text { DP) }\end{array}$ & $3 \pm 1,9$ & $3,5 \pm 3$ & $3,5 \pm 3$ \\
\hline \multicolumn{4}{|l|}{$\begin{array}{l}\text { Desfecho reprodutivo após } \\
\text { septoplastia }\end{array}$} \\
\hline Desejo de gravidez & 8 & 17 & 25 \\
\hline Gravidez & $2(25)$ & $14(82)$ & $16(64)$ \\
\hline Aborto precoce & - & $3(18)$ & $3(12)$ \\
\hline Gravidez extrauterina & - & $1(6)$ & $1(4)$ \\
\hline Parto pré-termo & - & $1(6)$ & $1(4)$ \\
\hline Parto de termo & $2(25)$ & $9(53)$ & $11(44)$ \\
\hline
\end{tabular}

DP: desvio padrão.
Tabela 2. Comparação dos desfechos reprodutivos prévios e após septoplastia

\begin{tabular}{lcc}
\hline & $\begin{array}{c}\text { Antes da septoplastia } \\
\text { n (\%) }\end{array}$ & $\begin{array}{c}\text { Após a septoplastia } \\
\text { n (\%) }\end{array}$ \\
\hline Aborto espontâneo & $17(61)$ & $3(12)$ \\
Gravidez ectópica & - & $1(4)$ \\
Parło pré-termo & $3(11)$ & $1(4)$ \\
Parło de fermo & $2(7)$ & $11(44)$ \\
\hline
\end{tabular}

\section{Discussão}

Após septoplastia histeroscópica obtivemos uma taxa de gravidez de $64 \%$ e uma taxa de recém-nascidos de termo de $44 \%$. Foi verificada uma melhoria do desfecho reprodutivo, com uma redução da taxa de aborto espontâneo de cinco vezes ( $61 \%$ antes da septoplastia e $12 \%$ após a septoplastia).

Atualmente não parece haver dúvidas de que os septos têm um impacto negativo na fertilidade. Contudo, os mecanismos etiopatogénicos não estão completamente esclarecidos, sobretudo no que diz respeito à relação entre septos e infertilidade primária. A hipovascularização septal (por hipoexpressão de RNA mensageiro dos recetores VEGF no septo), a placentação anormal e o aumento do tecido muscular com contratilidade anormal parecem interferir na implantação embrionária. Por outro lado, o déficit de recetores hormonais, a hiperpressão intrauterina por diminuição da cavidade uterina e a incompetência cervical são os mecanismos evocados para explicar o aumento de risco de aborto e o parto prematuro ${ }^{11-14}$.

Apesar da indicação clássica para a abordagem terapêutica dos septos ser os quadros de aborto recorrente, com o desenvolvimento de técnicas minimamente invasivas tem-se defendido a sua indicação para mulheres com antecedentes de abortos tardios, partos prematuros, infertilidade em nulíparas com mais de 35 anos, infertilidade inexplicada e previamente a técnicas de procriação medicamente assistida ${ }^{11,15,16}$. No presente estudo, 29\% das doentes tinham infertilidade primária, com uma idade média de 36 anos, e dessas, 88\% tinham um fator de infertilidade identificado, com predomínio dos fatores anovulatório e tuboperitoneal.

A septoplastia histeroscópica é atualmente a abordagem de eleição na correção de úteros septados ${ }^{17}$. Além da distensão da cavidade endometrial facilitar a secção da região central do septo, trata-se de um procedimento minimamente invasivo, associado a menor hemorragia e absenteismo laboral; que possibilita a conceção quatro a seis semanas após a sua realização e não implica na necessidade de parto por cesariana, comparativamente à septotomia por via abdominal ${ }^{11}$.

Quanto ao material cirúrgico utilizado na septoplastia histeroscópica, as microtesouras e eletrodos 
bipolares são atualmente os mais utilizados, apesar de múltiplos estudos randomizados controlados avaliando os diferentes resultados reprodutivos não demonstrarem diferença significativa relativamente à sua eficá$\mathrm{cia}^{11}$. Dois estudos randomizados demonstraram que a utilização de histeroscópio de $5 \mathrm{~mm}$ com eletrodo bipolar permite uma eficácia idêntica à da utilização do ressectoscópio monopolar. Considera-se, no entanto, que o primeiro apresenta mais vantagens, como menor risco de laceração cervical e de perfuração (não existe necessidade de dilatar o colo), menor duração do procedimento, menor risco da absorção do meio de distensão e de consumo de analgésicos ${ }^{18-20}$. No nosso estudo, por incluir doentes de um longo período de tempo, existe uma grande variedade de técnicas/materiais utilizados, inicialmente ressectoscopia monopolar, posteriormente ressectoscopia com ansa bipolar, e atualmente utilizando histeroscópio de $5 \mathrm{~mm}$ com ponteira bipolar ou microtesoura.

O procedimento deve ser idealmente programado para a primeira fase do ciclo, reservando para situações específicas a supressão endometrial (septos completos, espessos, endométrio espessado), sendo os agonistas de GnRH os mais eficazes. Não existem estudos randomizados comprovando ainda a eficácia da realização de antibioterapia profilática ou a necessidade de utilização de medidas para prevenir a formação de aderências (balão intrauterino ou terapêutica com estrogénios) ${ }^{11}$.

Uma metanálise de Nouri et al. ${ }^{21}$, incluindo 14 estudos com 1.324 mulheres submetidas à septoplastia histeroscópica, referia uma taxa de complicações de 1,7\%, sendo as mais frequentes hemorragia, sobrecarga hídrica, perfuração uterina, formação de aderências e rutura uterina numa gravidez subsequente ${ }^{11}$. No presente estudo confirmamos que a septoplastia histeroscópica é um procedimento rápido (média de 22 minutos) e seguro, tendo apenas ocorrido uma perfuração uterina (3\%). Apesar da elevada taxa de parto por cesariana na amostra (58\%), em nenhum dos casos a indicação da cesariana esteve associado à septoplastia, ou ocorreu rutura uterina.

No que diz respeito à necessidade de reintervenção, a metanálise de Nouri et al. ${ }^{21}$ referia uma taxa de
$6 \%$. No nosso estudo, essa taxa foi de $18 \%$, ocorrendo maioritariamente nas doentes com septo completo (em metade delas optou-se pela realização da cirurgia em dois tempos) de modo a minimizar a extensão do procedimento $^{1,21}$.

Apesar de haver inúmeros estudos publicados acerca do beneficio reprodutivo da septoplastia histeroscópica, não existem estudos randomizados controlados comparando a metroplastia histeroscópica e a manutenção de atitude expectante em mulheres com aborto recorrente, sendo esse um viés importante a apontar aos estudos publicados, incluindo o nosso ${ }^{22}$. Os resultados reprodutivos após septoplastia publicados na literatura variam consoante as populações avaliadas, contudo é no grupo das doentes com antecedentes de aborto que são mais significativos. $\mathrm{Na}$ metanálise de Nouri et al. ${ }^{21}$, foi verificada uma taxa de gravidez após tratamento de $60,1 \%$ e uma taxa de nativivos de $45 \%$. Numa metanálise de Valle e Ekpo ${ }^{11}$, a taxa global de gravidez após o procedimento foi de $67,8 \%$ e a taxa de nativivos de $53 \%$.

Os resultados obtidos no nosso estudo estão de acordo com a literatura, tendo-se obtido uma taxa global de gravidez de $64 \%$ e uma taxa de nativivos de $48 \%$, com redução significativa da taxa de abortos e aumento da taxa de partos de termo após a septoplastia histeroscópica.

O presente estudo tem algumas limitações, nomeadamente o número reduzido de casos e o fato de abranger um longo período de tempo, no qual foram utilizados diferentes materiais cirúrgicos. Foi ainda considerada apenas a primeira gravidez após o procedimento, entendendo os autores que essa é a que melhor reflete o impacto do procedimento cirúrgico.

Com o presente estudo podemos concluir que a septoplastia histeroscópica é um procedimento simples, seguro, eficaz e associado a uma baixa morbilidade. Verificamos ainda uma melhoria comprovada dos resultados reprodutivos com redução da taxa de aborto espontâneo de 5 vezes e uma taxa de nados vivos de $48 \%$. Assim, os autores reafirmam o lugar da septoplastia histeroscópica, como técnica minimamente invasiva de eleição, no tratamento de mulheres com útero septado e desfechos reprodutivos adversos.

\section{Referências}

1. Homer HA, Li TC, Cooke ID. The septate uterus: a review of management and reproductive outcome. Fertil Steril. 2000;73(1):1-14.

2. Moore KL, Persaud TVN. Embriologia clínica. 6a ed. Rio de Janeiro: Guanabara Koogan; 1998. p. 308-19.
3. Jacob M, Yusuf F, Jacob HJ. Development, differentiation and derivatives of the Wolffian and Müllerian Ducts. In: Yamada S, Takakuwa T, editors. The human embryo. Rijeka: InTech; 2012. p. 143-66. 
4. Massé J, Watrin T, Laurent A, Deschamps S, Guerrier D, Pellerin I. The developing female genital tract: from genetics to epigenetics. Int J Dev Biol. 2009;53(2-3):41 1-24.

5. Jaslow CR. Uterine factors. Obstet Gynecol Clin North Am. 2014;41(1):57-86.

6. Grimbizis GF, Camus M, Tarlatzis BC, Bontis JN, Devroey P. Clinical implications of uterine malformations and hysteroscopic treatment results. Hum Reprod Update. 2001;7(2):161-74.

7. Saravelos SH, Cocksedge KA, Li TC. Prevalence and diagnosis of congenital uterine anomalies in women with reproductive failure: a critical appraisal. Hum Reprod Update. 2008; 14(5):415-29.

8. Chan YY, Jayaprakasan K, Zamora J, Thornton JG, Raine-Fenning N, Coomarasamy $\mathrm{A}$. The prevalence of congenital uterine anomalies in unselected and high-risk populations: a systematic review. Hum Reprod Update. $2011 ; 17(6): 761-71$.

9. Venetis CA, Papadopoulos SP, Campo R, Gordts S, Tarlatzis BC, Grimbizis GF. Clinical implications of congenital uterine anomalies: a meta-analysis of comparative studies. Reprod Biomed Online. 2014;29(6):665-83

10. Carrascosa PM, Capuñay C, Vallejos J, Martín López EB, Baronio $M$, Carrascosa JM. Virtual hysterosalpingography: a new multidetector $\mathrm{CT}$ technique for evaluating the female reproductive system. Radiographics. 2010;30(3):643-61.

11. Valle RF, Ekpo GE. Hysteroscopic metroplasty for the septate uterus: review and meta-analysis. J Minim Invasive Gynecol. 2013;20(1):22-42.

12. Raga F, Casañ EM, Bonilla-Musoles F. Expression of vascular endothelial growth factor receptors in the endometrium of septate uterus. Fertil Steril. 2009;92(3): 1085-90.

13. Fedele L, Bianchi S, Marchini M, Franchi D, Tozzi L, Dorta M. Ultrastructural aspects of endometrium in infertile women with septate uterus. Fertil Steril. 1996;65(4):750-2.

14. Bendifallah S, Faivre E, Legendre G, Deffieux X, Fernandez $H$. Metroplasty for AFS Class V and VI septate uterus in patients with infertility or miscarriage: reproductive outcomes study. J Minim Invasive Gynecol. 2013;20(2):178-84.

15. Tomazevic T, Ban-Frangez H, Virant-Klun I, Verdenik I, Pozlep B, Vrtacnik-Bokal E. Septate, subseptate and arcuate uterus decrease pregnancy and live birth rates in IVF/ICSI. Reprod Biomed Online. 2010;21(5):700-5.

16 Ban-Frangez H, Tomazevic T, Virant-Klun I, Verdenik I, Ribic Puceli $M$, Vrtacnik-Bokal E. The outcome of singleton pregnancies after IVF/ICSI in women before and after hysteroscopic resection of a uterine septum compared to normal controls. Eur J Obstet Gynecol Reprod Biol. 2009; 146(2): 184-7.

17. Bosteels J, Kasius J, Weyers S, Broekmans FJ, Mol BW, D'Hooghe TM. Hysteroscopy for treating subfertility associated with suspected major uterine cavity abnormalities. Cochrane Database Syst Rev. 2013;1:CD009461.

18. Colacurci N, De Franciscis P, Mollo A, Litta P, Perino A, Cobellis $\mathrm{L}$, et al. Small-diameter hysteroscopy with Versapoint versus resectoscopy with a unipolar knife for the treatment of septate uterus: a prospective randomized study. J Minim Invasive Gynecol. 2007; 14(5):622-7.

19. Litta P, Spiller E, Saccardi C, Ambrosini G, Caserta D, Cosmi E. Resectoscope or Versapoint for hysteroscopic metroplasty. Int J Gynaecol Obstet. 2008;101(1):39-42.

20. Garuti G, Luerti M. Hysteroscopic bipolar surgery: a valuable progress or a technique under investigation? Curr Opin Obstet Gynecol. 2009;21(4):329-34.

21. Nouri K, Ott J, Huber JC, Fischer EM, Stögbauer L, Tempfer CB. Reproductive outcome after hysteroscopic septoplasty in patients with septate uterus: a retrospective cohort study and systematic review of the literature. Reprod Biol Endocrinol. 2010;8:52.

22. Kowalik CR, Goddijn M, Emanuel MH, Bongers MY, Spinder T, de Kruif JH, et al. Metroplasty versus expectant management for women with recurrent miscarriage and a septate uterus. Cochrane Database Syst Rev. 2011 ; 6:CD008576. 